\title{
Observations of Intermittent Structures in the Periphery of a Cylindrical Linear Plasma in PANTA
}

\author{
Noriko OHYAMA, Akihide FUJISAWA ${ }^{1,2)}$, Shigeru INAGAKI ${ }^{1,2)}$, Yoshihiko NAGASHIMA ${ }^{1,2)}$, \\ Stella OLDENBÜRGER ${ }^{2)}$, Tatsuya KOBAYASHI, Katsuyuki KAWASHIMA, \\ Yuuki TOBIMATSU, Tetsuo YAMASHITA, Soutaro YAMADA, Hiroyuki ARAKAWA ${ }^{3)}$, \\ Takuma YAMADA ${ }^{2,4)}$, Masatoshi YAGI ${ }^{2,5)}$, Naohiro KASUYA ${ }^{2,6)}$, Makoto SASAKI ${ }^{1,2)}$, \\ Sanae-I. ITOH ${ }^{1,2)}$ and Kimitaka ITOH ${ }^{2,6)}$ \\ Interdisciplinary Graduate School of Engineering Sciences, Kyushu University, \\ 6-1 Kasuga-koen, Kasuga, Fukuoka 816-8580, Japan \\ ${ }^{1)}$ Research Institute for Applied Mechanics, Kyushu University, 6-1 Kasuga-koen, Kasuga, Fukuoka 816-8580, Japan \\ ${ }^{2)}$ Itoh Research Center for Plasma Turbulence, Kyushu University, 6-1 Kasuga-koen, Kasuga, Fukuoka 816-8580, Japan \\ 3) Japan Atomic Energy Agency, 801-1 Mukoyama, Naka, Ibaraki 311-0193, Japan \\ ${ }^{4)}$ Graduate School of Frontier Sciences, The University of Tokyo, 5-1-5 Kashiwanoha, Kashiwa, Tokyo 277-8561, Japan \\ 5) Japan Atomic Energy Agency, 2-166 Oaza-obuchi-aza-omotedate, Rokkasho, Kamikita, Aomori 039-3212, Japan \\ 6) National Institute for Fusion Science, 322-6 Oroshi-cho, Toki, Gifu 509-5292, Japan
}

(Received 6 February 2012 / Accepted 16 February 2012)

\begin{abstract}
Intermittent pulse events have been observed in the periphery of the linear cylindrical plasmas produced in Plasma Assembly for Nonlinear Turbulence Analysis (PANTA). A novel combination of 32 azimuthal and 3 radial probes enables us to obtain $32 \times 3=96$ pairs of correlations simultaneously. The two-dimensional (2D) correlation analysis shows that the intermittent structure is well correlated with quasi-periodic bursts inside the plasma, and it reveals that the intermittent pulse is caused by the passage of a radially elongated and azimuthally distorted localized structure rotating in the ion diamagnetic direction.
\end{abstract}

(C) 2012 The Japan Society of Plasma Science and Nuclear Fusion Research

Keywords: two-dimensional correlation, multichannel probe, PANTA, intermittent structure

DOI: $10.1585 /$ pfr.7.1201025

In turbulent plasmas, many characteristic structures have been identified, such as zonal flows and streamers in turbulent plasma core $[1,2]$, blobs and intermittent phenomena in the plasma edge [3-7]. These structures are generated in turbulence to determine the final state of turbulence and the level of the turbulence transport. Therefore, the research on these structures and formation mechanisms has been highlighted as a topic of the modern plasma physics. In the linear cylindrical devices, PANTA and Large Mirror Device-Upgrade (LMD-U), a number of turbulent structures, such as zonal flows [8], streamers [9] and solitary waves [10], have been discovered and investigated with various kinds of multi-channel probes [9]. This article presents the observation of quasi-periodic intermittent pulse events in the plasma periphery of PANTA. The efficient $2 \mathrm{D}$-correlation analysis provides a new finding that the event is caused by the azimuthal rotation of a 'cloud' synchronized with periodic bursts inside the plasma, showing a similarity to the other phenomena such as avaloids [6] and coherent spiral structures [7] in 2D fast camera images.

The PANTA device produces a linear cylindrical plasma with a length of $4 \mathrm{~m}$ and a radius of $0.05 \mathrm{~m}$. The plasma is produced with helicon waves at $7 \mathrm{MHz}$ with a heating power up to $3 \mathrm{~kW}$. The device has been equipped with a number of multi-channel probes. The present results are obtained in Argon plasma at a magnetic field strength of $0.09 \mathrm{~T}$ and a relatively low filling gas pressure of 0.8 mTorr. With those discharge parameters, a quasiperiodic intermittent structure is observed in the plasma
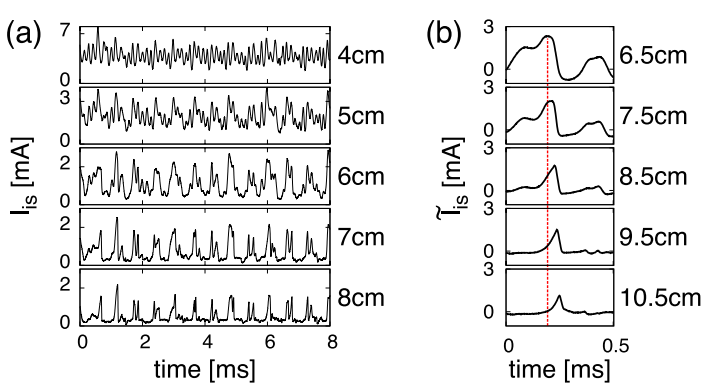

Fig. 1 A typical example of (a) time evolution of ion saturation current of five radially aligned probes at $r=4,5,6,7$ and $8 \mathrm{~cm}$, and (b) a pulse which looks to propagate radially at $r=6.5,7.5,8.5,9.5$ and $10.5 \mathrm{~cm}$. Horizontal axis in (b) is expanded to show the time delay more clearly. 

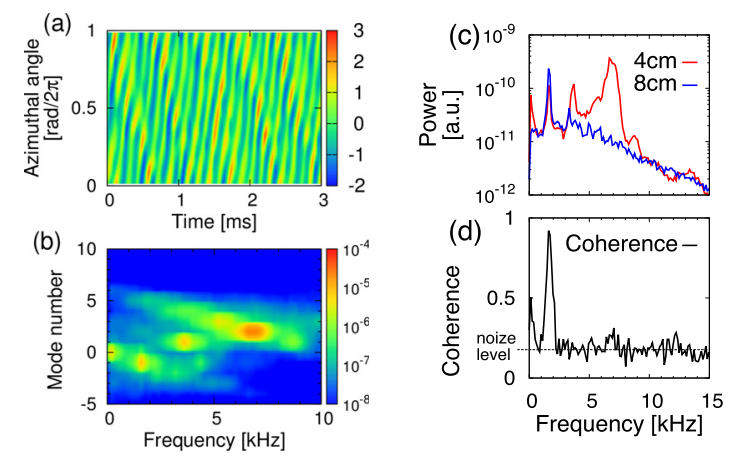

Fig. 2 (a) Ion saturation currents measured with 32 azimuthal probes at $r=4 \mathrm{~cm}$, (b) two dimensional spectrum of azimuthal mode number and frequency, (c) Fourier power spectra at $r=4 \mathrm{~cm}$ and $8 \mathrm{~cm}$, and (d) coherence between $r=4 \mathrm{~cm}$ and $r=8 \mathrm{~cm}$.

periphery. Figure 1 (a) shows a typical time evolution of the phenomena, simultaneously measured at five radial positions with a radial probe array; $r=4,5,6,7$ and $8 \mathrm{~cm}$. A zoom on a typical pulse event shown in Fig. 1 (b) suggests that a massive structure, like a blob, may propagate in the radial direction. Inside the plasma the fluctuations show turbulence-like or 'random' characteristics, while signals become pulse-like, and more structured, and the pulse width becomes narrower in outer radii (say, $r=8 \mathrm{~cm}$ ). Correspondingly, the change of the characteristics is clearly shown by the probability density function (PDF) analysis: a quasi-Gaussian distribution in the core changes to a non-Gaussian one at $r \sim 6 \mathrm{~cm}$.

The quasi-Gaussian property inside the plasma is realized by the coexistence of several characteristic modes in the spectrum shown in Fig. $2 ;$ e.g., $(f, m)=(1.6 \mathrm{kHz},-1)$, $(3.6 \mathrm{kHz}, 1),(6.8 \mathrm{kHz}, 2)$ and $(5.6 \mathrm{kHz}, 3)$, where a negative azimuthal mode number means that the mode propagates in the ion diamagnetic direction. Figures 2 (a) and 2 (b) show the periodic bursts and its power spectrum in frequency and wavenumber domain, respectively. The nonlinear couplings between such coherent modes shown in Fig. 2(b) were confirmed to produce the quasi-periodic phenomenon in LMD-U [9]. Figure 2 (a) shows that the structure with an azimuthal size of $0.3 \pi$ radian, appears to be propagating in the electron diamagnetic direction locally and then disappears in a local lifetime of $\sim 0.1 \mathrm{~ms}$. The structure then appears again in the neighboring azimuthal position separated by $\sim 0.3 \pi$ radian from the previous position in the ion diamagnetic direction.

Cross-power analysis is performed on one of 32 azimuthal probes located at $r=4 \mathrm{~cm}$, with one movable probe that scans the range of $r=2.5-11 \mathrm{~cm}$. Spectral characteristics are quite different between the inside and outside (see Fig. 2 (c)), the larger and weaker (but significant) coherences are found at the frequencies of the modes of $m=-1$ and $m=2$, respectively; the power of $m=-1$ at $1.6 \mathrm{kHz}$ is still high, while that of $m=2$ at $6.8 \mathrm{kHz}$ is
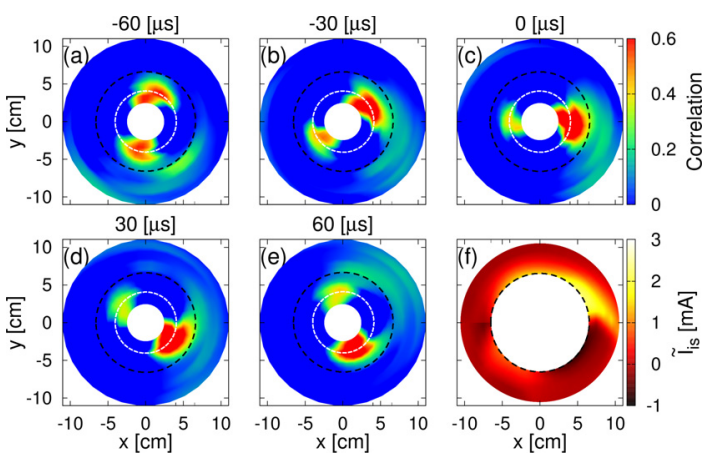

Fig. 3 Temporal evolution of correlation function $C(\Delta r, \Delta \theta, \tau)$. (a) $\tau=-60 \mu \mathrm{s}$, (b) $\tau=-30 \mu \mathrm{s}$, (c) $\tau=0 \mu \mathrm{s}$, (d) $\tau=30 \mu \mathrm{s}$, (e) $\tau=60 \mu$ s. Note that all negative values are shown by the same color as 0 in the figures. (f) Reconstructed image of a spatial structure of a pulse event. White and black dotted lines indicate the position $r=4 \mathrm{~cm}$ and $6.5 \mathrm{~cm}$, respectively.

no longer visible outside (Fig. 2(d)). The spatio-temporal evolution of the structure can be deduced by evaluating the $2 \mathrm{D}$ correlation between 32 probes of the azimuthal probe array and 3 probes with each radial distance of $5 \mathrm{~mm}$.

Using these probes, $32 \times 3=96$ pairs of correlations can be analyzed simultaneously. Assuming that every azimuthal probe should be identical as a reference due to the symmetric nature of a cylindrical plasma, the 2D correlation covering both radial and azimuthal dimensions with $\delta r=1.5 \mathrm{~cm}$ and $\delta \theta=360$ degrees can be obtained at a single shot. The temporal evolution of the obtained 2D correlation function, $C(\Delta r, \Delta \theta, \tau)$, is shown in Fig. 3 and provides a statistical idea of how the intermittent pulse-like structure should evolve, where $\Delta r, \Delta \theta$ and $\tau$ denote the radial distance, azimuthal angle from a reference probe and time lag, respectively.

The correlation shows that, in the inner region of $r=6 \mathrm{~cm}$, a structure with $m=2$ rotates in the electron diamagnetic direction. In the outer region of $r=6 \mathrm{~cm}$ an $m=1$ structure, like a cloud, rotates oppositely in the ion diamagnetic direction, with the angular velocity of $(1.25 \pm 0.25) \times 10^{4} \mathrm{radian} / \mathrm{s}$ constantly at every radius in the periphery. This value is almost consistent with the velocity of an $m=1$ structure at $r=4 \mathrm{~cm}$. Therefore, the pulse-like structure observed in the radial probes should reflect azimuthal structure of the cloud when it is passing the probes. The delay between the peaks of the pulses should be caused correspondingly by the azimuthal distortion of the cloud structure.

Based on the assumption that the rigid-body structure rotates with a constant angular frequency $\omega$ in the ion diamagnetic direction, the structure can be reconstructed using the relation, $I(r, \theta, t)=I(r, \theta-\omega \tau, t-\tau)$. Figure 3 (f) shows an example of such reconstruction for the case shown in Fig. 1 (b). In real situation the assumption cannot be fully valid. The really created structure should 
be different in each event, which should cause the statistical broadening, or the cloud-like structure, of the 2D correlation. In order to confirm the real evolution and capture the actual characteristics of the structure, 2D measurements covering the whole region where the phenomenon occurs are essentially necessary.

This work is partly supported by a Grant-in-Aid for Scientific Research from JSPS (21224014, 23246162, 23360414, 23360409), and the collaboration programs of RIAM Kyushu University and NIFS (NIFS10KOAP023).
[1] A. Fujisawa, Nucl. Fusion 49, 013001 (2009).

[2] S.-I. Itoh, J. Plasma Fusion Res. 86, 334 (2010).

[3] D.A. D'lppolito et al., Phys. Plasmas 18, 060501 (2011).

[4] S.H. Müller et al., Plasma Phys. Control. Fusion 51, 055020 (2009).

[5] T. Windisch et al., Phys. Plasmas 13, 122303 (2006).

[6] G.Y. Antar et al., Phys. Plasmas 14, 022301 (2007).

[7] H. Tanaka et al., Contrib. Plasma Phys. 50, 256 (2010).

[8] Y. Nagashima et al., Phys. Plasmas 16, 020706 (2009).

[9] T. Yamada et al., Nature Phys. 4, 721 (2008).

[10] H. Arakawa et al., Plasma Phys. Control. Fusion 53, 115009 (2011). 